\title{
Physical and biological bases of laser phototherapy
}

\author{
Jan Rykała ${ }^{1}$, Paweł Szychta ${ }^{1}$, Henryk Witmanowski2,3 \\ 1Department of Plastic, Reconstructive and Aesthetic Surgery, Medical University of Lodz, Poland \\ Head: Prof. Bogusław Antoszewski MD, PhD \\ 2Department of Plastic, Reconstructive and Aesthetic Surgery, Nicolaus Copernicus University in Torun, \\ Collegium Medicum in Bydgoszcz, Poland \\ Head: Prof. Henryk Witmanowski MD, PhD \\ 3Department of Physiology, Poznan University of Medical Sciences, Poland \\ Head: Prof. Teresa Torlińska MD, PhD
}

Postep Derm Alergol 2012; XXIX, 5: 378-383

DOI: $10.5114 /$ pdia.2012.31492

\begin{abstract}
The authors present physical properties of laser light, operating principles, kinds of lasers and also physical and biochemical bases of biological laser light action on human cells, tissues and living organisms. The authors discuss a broad range of biological mechanisms of laserotherapy, which were examined on different levels (cell, tissue, organ) and which let them explain the positive influence of laser light in different diseases. Currently known therapeutic effects of lasers may be in many cases beneficial, allowing for effective rehabilitation of the chronically ill, the possibility of replacing hospital-based surgery with outpatient conservative treatment (which is significantly shorter and the costs are reduced), as well as creating opportunities for the treatment of diseases as yet incurable.
\end{abstract}

Key words: laser light, laserotherapy, physical and biochemical bases of biological laser light action on human cells.

\section{Introduction}

The word "LASER" is an acronym formed by the first letters of words of the English phrase "Light Amplification by Stimulated Emission of Radiation". Laser radiation is an optical amplifier; its operation is possible thanks to the phenomenon of stimulated emission. This phenomenon was predicted theoretically and announced by Albert Einstein in 1917 [1]. The amplifier can become a generator after the feedback in specific conditions. Today, the term "laser" refers to electromagnetic radiation generators operating in a wide range of the electromagnetic spectrum from microwaves to X-rays. The first laser was a ruby laser, launched by Maiman in 1960. This laser generates pulses of coherent light of a wavelength of 694.3 nm (red) [2]. Another laser was a helium-neon laser, built by Javan in 1961 [3]. This machine generates a continuous laser beam with a length of $1150 \mathrm{~nm}$ (near infrared). In Poland, the first laser was built in 1963. It was a helium-neon laser, run by a team of Professor Zbigniew Puzewicz at the Military University of Technology [4, 5].

\section{Physical properties of the laser radiation}

The importance of constructing the first lasers is associated with unusual properties of the generated radiation, as compared with the radiation obtained from the existing light sources. Light from the laser is different from the light emitted by classical sources, similarly to the difference between music and noise. The characteristic features of laser radiation result from the properties of the phenomenon of stimulated emission [4, 5]. Quanta emitted in the process of this emission and the forcing quanta have the same frequency, direction of propagation, phase and polarization. Light from a laser is a collection of the same basic portions of energy (photons) as the ordinary light, but the wave picture of light photons in the laser exhibits a significantly higher degree of order. The distinguishing feature of laser light sources from the classical ones is a much higher spectral density of the emitted radiation. Unlike conventional light sources, the laser sources radiate directionally. The angle of divergence of the laser beam depends on the shape of the resonator, but usually it does not exceed a few milliradians. 
Thus, the laser energy is concentrated in a very narrow range of frequencies and a small solid angle.

In the laser the conditions can be created for pulse generation of nano-, pico- and femtosecond recently, allowing to work with a power up to $18 \mathrm{~W}$. Wavelength of the radiation generated by the laser depends on the active medium, i.e. the medium in which the phenomenon of enhancing the light may occur as a result of stimulated emission [4-7].

\section{Types of lasers}

Since Javan and Maiman launched the first lasers, several devices that generate from ultraviolet radiation (excimer laser ArF - $193 \mathrm{~nm})$ to the infrared $\left(\mathrm{CO}_{2}\right.$ laser $1060 \mathrm{~nm}$ ). Due to the physical state of the active medium, lasers are divided into solid, liquid, gas and solidstate. Representatives of solid lasers include the ruby laser $(694.3 \mathrm{~nm})$ and neodymium one (1064 nm). The liquid lasers are tunable such as a dye. The active part of gas lasers are usually a mixture of gases or vapors in a closed discharge pipes. Due to the fact that active components in gas lasers, such as atoms, ions or molecules can be used in the generation process, these devices are still often divided into atomic lasers (helium-neon laser 632.8 $\mathrm{nm}, 1150 \mathrm{~nm}$ ), ion (Argon laser 488-514 nm), molecular $\left(\mathrm{CO}_{2}\right.$ laser $\left.10600 \mathrm{~nm}\right)$ and a metal vapor laser $(\mathrm{Au}-628 \mathrm{~nm}$, $\mathrm{Cu}-578 \mathrm{~nm}$ ). Semiconductor lasers form a separate group in this division. The active element is a solid, and the strengthening and generation of coherent radiation occurs near the connector of a semiconductor of type "p" with a semiconductor of type "n" (GaAlAs 780-1100 nm).

Due to the generated wavelength lasers are divided into: UV lasers (excimer ArF - $193 \mathrm{~nm}$ ), visible lasers (He-Ne 632.8 nm, Ar 488-514 nm, Rb 694.8 nm) and infrared laser (Nd: YAG $1060 \mathrm{~nm}$, GaAlAs 780-1100 nm, $\left.\mathrm{CO}_{2} 10600 \mathrm{~nm}\right)$.

In medical applications often, similar to X-rays, lasers are classified due to the average and continuous emitted power. According to this criterion, lasers are divided into: soft - up to $5 \mathrm{~mW}$, medium - up to $500 \mathrm{~mW}$, and hard with a capacity of more than $500 \mathrm{~mW}$.

Each laser can be used therapeutically. Therefore, the often used term "medical laser" is not accurate, because you could extract any kind of laser that could have such an application. Today, lasers are used in all medical specialties to treat a wide variety of diseases, which is allowed by a huge variety of hardware and variety of its physical interactions $[2,7]$.

\section{Physical mechanisms of interaction between laser light and the living matter}

The mechanisms responsible for the effects of laser radiation with the living matter are fundamentally different from the mechanisms of laser interaction with inan- imate physical objects. They depend on the parameters characterizing the laser beam, like power, wavelength, pulse duration or exposure time and cross-sectional area of the incident beam. They also depend on the parameters characterizing the biological object illuminated such as absorption and scattering coefficient, which depends on the used wavelength, density of the medium, the specific heat and coefficient of thermal conductivity. In addition, an important role in the living organism is played by the flow of blood and lymph, by which heat transfer occurs from the area of interaction of laser light with tissue $[2,3,8]$.

For example, in the interaction of laser radiation to the tissue in the infrared range, the radiation energy is converted into rotational and oscillatory energy of molecules; the visible light excitation is external electron; and ultraviolet radiation - internal electron excitation. Thus, interactions of laser light with the living matter are affected by numerous factors, many of which (much higher ratio than it is for physical objects) depends on the parameters of a biological object $[3,9,10]$.

\section{Biological effects of laser light with the living matter}

Therapeutic effect is desirable and it is the most awaited by doctors. Its core is the physico-chemical interaction of the laser beam on the biochemical structure of the cell, so that its properties are changed and as a consequence - their activity. These changes, initially only qualitative, with the increasing current and power of the laser beam may have destructive effects, which are initially reversible, then irreversible. Each type of these changes may be a treatment effect in certain circumstances. The interaction of laser radiation with tissues can be grouped into three categories: 1 - photothermal effects, 2 - photoionizing impact, and 3 - photobiochemical impact.

Photothermal effects occur at power densities above $1 \mathrm{~W} / \mathrm{cm}^{2}$ and at the impact of time longer than a nanosecond. With these parameters we can get radiation denaturation, coagulation, hemostasis, cutting, welding, tissue ablation, etc. The faster the energy is delivered to the tissue, the less impact on the surrounding tissues. For precise cutting we need high power density and short radiation. At a lower level of power and intensity of the laser, the thermal effect also occurs because it is always a result of laser light providing the extra energy. If this energy is low and spread over an appropriate time, the thermal effect does not exceed the compensation capacity of the cell. As the energy supplied increases the thermal effect can dominate the effect of the biochemical in a cell but can be compensated at a level of the tissue. A further increase of thermal energy causes destruction beyond the capacity of compensatory tissue and can lead to its destruction, it can still be aligned at the level of the organ. 
The increase in kinetic energy of the molecules, resulting from the thermal effect, for example, can lead to changes in the permeability of cell membranes, the cytoplasmic membranes surrounding organelles, and thus to changes in the activity of enzymatic systems and, in addition to changes in the intracellular and intercellular transport.

At the same power density with nanosecond irradiation times and shorter, we will observe the photoionizing effects, including the photo-breaking bonds. Frequently photoionization leads to changes in the electrical system of particles of organic compounds (proteins and enzymes) which may cause the change of their activity, and thus changes in cell metabolism. Changes in the electric charge within the cell membrane cause changes in permeability as well as in the conductivity, such as in nerve cells. However, photo-cleavage of bonds may lead to the formation of new chemical compounds and therefore may significantly alter cellular metabolism.

Photobiochemical effects occur at power densities up to $1 \mathrm{~W} / \mathrm{cm}^{2}$ when the dwell time is relatively long, such as microseconds or longer. These parameters of radiation are used in biostimulation.

The photobiochemical impact, according to the state of our knowledge, can be distinguished to two different mechanisms in its essence. These are: a photochemical effect and biochemical resonance effect.

Photochemical effect - consists of selective and specific responses of natural dyes found in tissues with specific lengths of electromagnetic radiation. These dyes are known, not very precisely as photosensitizers. These include for example hemoglobin and melanin. The main photosensitizer, from the point of view of the evolution, is chlorophyll, which "action" is started with a wave of radiation in the ultraviolet range. A good example of the photochemical action of light is the mechanism of vision activated with the visible light radiation in only $300 \mathrm{~nm}$ range (from $400 \mathrm{~nm}$ to $700 \mathrm{~nm}$ ), causing the color distribution of the proteins in the retinal pigment epithelium. Photosensitizers are enzymatic systems (for example, cytochromes), and they also become several different waste products (such as precursors and derivatives of hemoglobin). It is possible that in some diseases several blood proteins take also photosensitizers property. This may explain the experimentally observed entirely different response to the same length of the laser light of the healthy and the sick bodies. There is also a group of photosensitizers obtained synthetically, which may cause very different effects than the effects of laser light generated without their presence, depending on where they accumulate in tissues.

Biochemical resonance effect - is the "forcing" the spatial reorientation of the particles of organic compounds (such as enzymes) by uniform oscillation of the laser light, and thus a significant increase in their biological activity. A good example of this resonance imaging is the design of the bridge. It can sustain the highest traffic, provided that each of the moving objects makes its own unique rhythm of vibrations with different frequencies and with different intensity. They mute one another and the construction of the bridge takes over the excess of the vibration and suppresses its inertia. However, a small branch of the military going through the same bridge, but step marching, puts the same design in increasing vibration, enforced by the rhythmic, consistent, and uniform vibration of this "external object". This leads to the inevitable collapse of the bridge if the resonance of the vibration is sufficiently long. Biochemical effects resulting in cell structures under the influence of laser light changes depend on the spatial orientation of organic molecules under the influence of specific frequency of light energy and an increase of organic and inorganic molecules, which is the essence of a thermal effect. The first of these mechanisms can lead to an increase in the activity of individual enzymes or enzyme systems in the whole of the organelles that are natural resonator of specified wavelength (frequency of oscillation). Respiratory enzymes can be activated in the mitochondria or digestive enzymes in the lysosomes.

Diagram presented below illustrates the possibilities of obtaining a various biological effect in the same cell, depending on the healthy/sick physiological state from organelle "absorbing" laser radiation, in presence or absence of a photosensitizer, when using only a single source of laser radiation, where the "+"presupposes a specific organelle function,"-"indicates a decrease in its activity, " $\mathrm{O}_{2}$ " means the effect of oxidation and "R" means reducing effect.

\section{Diversity of the biological impact of laser radiation}

Impact mechanisms of laser light on the living tissue depend on the one hand, on its physical properties, as mentioned above, on the other hand - the type of tissue, the degree of differentiation of cells building the given tissue and physiological state of organelles in individual cells. Effect of laser light on the body is therefore the result of the physical properties of the laser light and the physicochemical properties of the cell, which receives the light.

The interference of laser radiation begins in the living body in the molecular level. Changes in this level affect activity of the specified cell structures (e.g. organelles), and subsequently the change of cell properties and, consequently, change in the properties of the tissue. This leads to a change in a particular organ, and eventually to the changes in the body.

In the case of biochemical effects dominating over thermal effects we can talk about laser stimulation, in the opposite case - the dominance of thermal effects on biochemical - we have a coagulating effect.

Exposures of the biologically diverse types of tissues to different physical properties of laser light, we have to 
deal with different compositions of these changes, which in any case can produce quite different effects of the new behavior of irradiated tissue.

In a relatively numerous and varied experiments on laboratory animals and tissue culture using a variety of laser light, generated biological effects can be seen in the form of numerous changes [2, 3, 7, 8]. The irradiated tissue has increased blood flow, which enables a quicker exchange of electrolyte between the cells. Improved tissue perfusion is also the result of enhancement of angiogenesis, which occurs as early as 5 days after the beginning of exposure.

Laser radiation in the infrared range increases the diameter of the lymphatic vessels, causing a greater flow of lymph and accelerates regeneration after cutting, activation of bone marrow and the increased number of red blood cells with their enhanced osmotic resistance. It has immunosuppression effect, as evidenced by a decrease in the activity of $T$ cells and reduced production of antibodies, while the wound healing has been noticed to have increased phagocytic activity of monocytes and neutrophils. Laser radiation in the range of red light affects the dynamics of the formation and removal of strontium antigens and antibodies in the rabbit cornea caused by the Arthus reaction. Helium-neon laser radiation stimulates the production of cytokines, such as interleukin- $1 \alpha$, interleukin-2 and interferon- $\gamma$, with the unaffected expression of the surface cell receptor for interleukin-2. Inhibition of increased permeability of blood vessels was observed, together with increase in swelling in the acute phase of inflammation and the formation of granulation tissue.

An increase in the concentration of adrenaline and noradrenaline in both irradiated wounds, blood and the hypothalamus of experimental animals, as well as changes in the concentration of histamine and serotonin, under the influence of laser radiation. Changes in the concentration of substances such as histamine and serotonin are associated with analgesic action of laser radiation. Decreased sensitivity to pain was maintained up to 7 days after the end of the exposure cycle.

The cell cultures were observed for intensification of resilience, which refers mainly to the connective tissue and epithelial cells; expression of this is the rapid healing of wounds, burns, bone fractures. The main mechanism for better recovery is accelerated cell proliferation, which in turn is made possible by the increase their metabolism.

There are single reports of laser radiation to facilitate the transition of very well differentiated tissue cells from the stationary phase into the mitotic cycle. Increased transcription of genes of many proteins was noted, particularly clear in the case of collagen.

Cartilage being connective tissue responds to laser irradiation with increased matrix synthesis, starting on day 6 of exposure. Radiation of helium-neon laser has a stimulating effect on the regeneration of skeletal muscle in the rat and the faster maturation.

Laser radiation also affects the denervated muscles. Inhibition of changes characteristic for the denervation of muscle growth was seen, such as reduction in acetylcholine receptors and phosphokreatynine kinase level. Laser radiation in the range of ultraviolet, visible and infrared light causes miorelaxation of vascular smooth muscle. Laser radiation increases the functional potential of the not damaged peripheral nerves and nerves that have been crushed; in the zone of injury it accelerates the growth of nerve fibers and their mineralization. Laser radiation also inhibits degenerative processes of spinal motoneutrons corresponding to the damaged nerve.

In the case of embryonic neural tissue grafts to the spinal cord or the brain of experimental animals, it inhibits the formation of the exuberant glial scar, which is usually an obstacle to the final survival of the transplant. It has been shown that the increase in neurite outgrowth from embryonic neuronal cells under the influence of laser radiation is accelerated.

Laser radiation influences on the division of Schwann cells, specialized cells which pill up along the axon outgrowth and are involved in the production of the myelin sheath. The increasing growth rate of bacteria was observed, and their physiological activity is intensified under the influence of appropriately selected conditions of exposure. It is noted that the change rate of cell division is dependent on the wavelength of the radiation energy per unit area, length of exposure, and also the functional state of the cells. The impact of this radiation on keratinocytes is not clear. It was because of accelerated cell proliferation, which was manifested in three times faster moving edge at the point of damage due to infrared laser radiation, the effect is dependent on the density of the radiation energy. These results are crucial in explaining the beneficial effects of laser radiation in the treatment of leg ulcers.

In the experiment concerning the human lymphocytes from the peripheral blood in nuclear chromatin, the effect is similar to blast transformation induced by phytohaemagglutinin. After exposure, there was reversible, increased influx of calcium ions into cells, increased binding of acridine orange to DNA and reconstruction of the ultrastructure of nucleoli. This would indicate the transiently increased cell membrane permeability to calcium ions, stimulating the nuclear chromatin and increased transcription of ribosomal DNA. Assessing the quantitative and qualitative changes in the mitochondria of the irradiated cells, an increase in the number of cells without increasing the surface area and total weight was seen.

Fibroblasts, cells that play a central role in the healing process, respond to radiation He-Ne laser with functional and structural changes, increasing collagen and DNA synthesis and widening of endoplasmic reticulum. 
These changes were not accompanied by degenerative changes in cellular organelles. Laser radiation at the same wavelength converts fibroblasts to myofibroblasts already $24 \mathrm{~h}$ after irradiation. Increasing the population of fibroblasts equipped with retractable elements may be important in the formation of scar tissue morphology and to improve its mechanical strength, especially after surgery.

A study concerning oocytes of Chinese hamster and human fibroblasts revealed that most mutagenic is ultraviolet excimer laser. Human fibroblasts irradiated with light of green argon laser did not result in any changes in the genetic material, allowing to conclude that the laser radiation used in biostimulation showed no mutagenic effect. Moreover, the experimental data seem to suggest its antimutagenic action. The study was performed on embryonic human cells and measured the number of chromosomal mutations due to neutron or $\gamma$ radiation. After application of ionizing radiation the cells were exposed for 30 min to helium-neon laser radiation. In cells exposed to neutrons flux, the number of mutations increased fivefold. Laser radiation caused no increase in the number of mutations. In the group of cells subjected firstly to the action of neutron flux, and then the laser, the number of mutations was low, similar to the control group. The same reaction was demonstrated in the case of $\gamma$ radiation. This effect can be explained by the influence of the laser, which stimulates the DNA photoreactivation enzyme. Similar results were obtained with chemical mutagens, such as acridine.

Changes in the structure and surface properties of the membranes were seen in erythrocytes and platelets, neurons, mitochondria, and many other cells and organelles. For all measuring frequency the conductivity of blood in irradiated animals was significantly increased compared with the control group. The obtained data show that prolonged exposure to low-energy laser radiation causes changes in the electrical properties of the blood of experimental animals, possibly due to changes in membrane properties of erythrocytes. there are changes in the membranes in the concentration of acidic phospholipids fractions, changes in permeability and conductivity as well as adhesion properties and surface electric charge of the membrane. This amounts to less aggregation of erythrocytes, better transport of oxygen, modification of the oxidative phosphorylation in the mitochondria and altered neuronal action potential [10].

Laser radiation affects the DNA content in cells by enhancing its synthesis. The result of this phenomenon is enhanced by increased cell proliferation and protein synthesis. Under the influence of He-Ne laser there are major changes in the structure of mitochondria. The morphological difference is the dichotomy or small sizes with high transparency matrix. Other phenomena occurring in irradiated mitochondria are changes in their optical properties, i.e. the absorption and emission spectra, which clearly demonstrates a direct interaction of laser radiation with these organelles. There was also an increase in the electrochemical gradient across the mitochondrial membrane, as well as the activation of mitochondrial genetic apparatus, which is reflected by the increased synthesis of DNA and RNA by almost 100\% and which is the consequence of increased protein synthesis.

An important phenomenon is an increase in ATP with the simultaneous increase in the phosphorylation rate and reduction in the demand for oxygen. The liver cells were treated with irradiation increased by almost 30\% of the stock of ATP and more active oxidation-reduction systems. A study on tissues, cells or cell homogenates showed that the activity of most dehydrogenases, transaminases, hydrolases increases under the influence of laser radiation by about $20 \%$. However, exposure of isolated enzymes in vitro leads to no increase of their activity.

Different groups of enzymes (oxidoreductases, transferases, hydrolases) which are inactivated by factors such as high temperature, carbon dioxide, low pH, go back to the initial activity by using the laser radiation of the range of red light and infrared light [9].

Interesting experimental data were obtained in the last few years studying the effects of low power laser radiation on non-enzymatic structural proteins. The laser He-Ne causes the transformation of skeletal structure in heteroploidal human cells after $15 \mathrm{~min}$. Microtubules are becoming more numerous and form a more dense network of centers of aggregation. Intermediate filaments (keratin, wimetin) are also numerous forming a thick ring around the nucleus. Microfilaments (actin, myosin) form thicker fibers. Interesting is the fact that similar transformations are microfilaments in plant cells responsible for the redistribution of chloroplasts depending on the direction and intensity of light. Twice the tensile strength of the tendons was detected which heal after cutting with help of helium-neon laser. Furthermore, using electron microscopy, it was found that tropocollagen fibers in the irradiated tendon are more uniformly aligned along the long axis [11].

As is clear from the above list of observed biological changes induced by the laser radiation, the diversity of these effects is huge. It should be remembered that as presently as throughout the relatively short history of medical uses of lasers (history of the laser is only 40 years old!) clinical practice is ahead of basic research. Usually methods of radiation first appear to be effective in different disease entities, and after a considerable delay they are studied and only fragmentary, biological mechanisms responsible for the specific therapeutic effect are usually found. At present there are more questions than answers. This raises the need to mobilize the full basic research in the field of laser light interacts with living matter. 


\section{Conclusions}

Currently known therapeutic effects of lasers may be in many cases beneficial, allowing for the effective rehabilitation of the chronically ill, the possibility of replacing hospital-based surgery with outpatient conservative treatment (which is significantly shortened and the costs are reduced), as well as creating opportunities for the treatment of diseases as yet incurable.

\section{References}

1. Trainer M. The 50th anniversary of the laser. World Pat Inf 2010; 32: 326-30.

2. Sroka R, Lilge L. Medical laser applications and laser-tissue interactions. Med Laser Appl 2010; 25: 264.

3. Ellis JD, Voigt D, Spronck JW, Verlaan AL, Munnig Schmidt RH. Frequency stabilized HeNe gas laser with $3.5 \mathrm{~mW}$ from a single mode. Prec Eng 2010; 36: 203-9.

4. Belkin M, Schwartz M. New biological phenomena associated with laser radiation. Health Phys 2005; 5: 687-90.

5. Kaczmarek F. W ćwierćwiecze odkrycia lasera. UAM Poznań 1987, Fizyka 55.

6. Salehinia D, Khorasani K, Behrouzinia S, Zand M. Small-signal gain dependence on active medium length and axial thermal profile in metal-vapor lasers. J Russ Laser Res 2011; 32: 68-73.

7. Carroll L, Humphreys TR. LASER-tissue interactions. Clin Dermatol 2006; 24: 2-7.

8. Lou WW, Geronemus RG. Dermatologic laser surgery. Semin Cutan Med Surg 2002; 21: 107-28.

9. Soldatov A, Reimer IV, Evtushenko VA, Melnikov KY, Malikov AV. Tunable wavelength laser medical system for treating oncological diseases. Bull Lebedev Phys Inst 2010; 37: 4-5.

10. Sadighha A, Mohaghegh Zahed G. Meta-analysis of hair removal laser trials. Lasers Med Sci 2009; 24: 21-5.

11. Joensen J, Gjerdet NR, Hummelsund S, et al. An experimental study of low-level laser therapy in rat Achilles tendon injury. Lasers Med Sci 2012; 27: 103-11. 